\title{
Study of the surface structure of Fe quantum wire prepared by electrochemical method in solution
}

\author{
Zhaoyun $\mathrm{Yu}^{\mathrm{a}}$, Yong Xia ${ }^{\mathrm{b}}$, Xiaodong Dong ${ }^{\mathrm{a}, *}$ \\ ${ }^{a}$ Medical College, Hebei University, Baoding 071002 China \\ b State Key Laboratory of Electroanalytical Chemistry, Changchun Institute of Applied Chemistry, Chinese \\ Academy of Sciences, Changchun 130022 China
}

*Corresponding author, e-mail: xddong@hbu.edu.cn

Received 6 Jul 2020

Accepted 18 Oct 2020

\begin{abstract}
The surface structural characteristics of Fe quantum wires prepared by two electrochemical methods in solution were studied by their conductance changes caused by the interactions between the quantum wires and specific molecules. Once ascorbic acid was added into the solution, the conductance of the Fe junction decreased to a lower value while for the dopamine adsorption, the conductance showed no obvious change. We proposed that there were some iron(II) ions on the surface of atomic-scale Fe junction, not iron(III) ions, and these iron(II) ions played a significant role for the conductance change. This mechanism was further enhanced by the following two experiments. When exposing the quantum wire to sodium borohydride, the conductance increased to a higher value, and the conductance increase was also observed with an electrochemical reduction process, which provided additional evidence for the proposed mechanism.
\end{abstract}

KEYWORDS: iron, surface, magnetic materials, electrochemistry, quantum wire

\section{INTRODUCTION}

When the size of the materials and device is reduced to nanometer scale, many novel quantum phenomena will appear, which will bring many new and important applications [1-3]. A typical case is the quantum conductance behavior of the atomically thin metal junction, which has been verified with a large number of theoretical and experimental studies in the past decades [4-6]. If the width of a metal wire reaches its Fermi wavelength scale, the conductance will not change continuously with the diameter, and it will be quantized by $G=$ $G_{0} \sum_{i=1}^{n} T_{i}$ where $G_{0}=2 \mathrm{e}^{2} / h$ (e and $h$ represent, respectively, the electron charge and Planck's constant, so $G_{0} \approx 1 / 12.9 \mathrm{k} \Omega$ ), and $T_{i}$ represents the transmission coefficient for each quantum mode. For ideal metal wires like Au and Ag, the value of $T_{i}$ is approximately equal to 1 , and thus the electrical conductance passing through the atomically thin metal junction is given by $G=N G_{0}$, where $\mathrm{N}$ is an integer. Thus, it shows an integer multiple change of $G_{0}$, quantum conductance. The atomically thin metal wire (junction) is also known as a metal quantum wire. Because the width of the wires could be compared with the Fermi wavelength of conducting electrons in the metals, they will have many novel properties different from their bulk materials such as photic, electrical, magnetic and surface properties.

Although the metal quantum wires have received much attention for the last decades due to their potential applications in nanoelectronics and chemical sensing [7-9], few studies have been reported on the surface microstructures of this novel nanostructured material, especially in aqueous solution. Because the cross section of a metal junction is composed of only a few atoms and most atoms are located on the surface of the junction, distinctive characteristics unlike those of bulk form can be expected. Moreover, understanding the interfacial properties of molecules in molecular electronics is an important task. Therefore, exploring the surface microstructural characteristics of the atomically thin quantum wires and understanding their surface and interface behaviors are very meaningful in many scientific fields such as surface and interface science, nanoelectronics and sensing.

Our group [10] has previously studied the interactions between silver quantum wires prepared by an electrochemical method and ammonia molecules in aqueous solution. Based on the conductance change of the atomic scale silver junction, we proposed that there were some silver ions on the surface of silver quantum wires. Today, as an important 
member of one-dimensional magnetic materials, the iron-based nanostructured materials not only show the superior magnetic properties, but also draw extensive attention in biomedical applications owing to the wide application prospects in medical diagnosis and treatment, resulting from the high saturation magnetism and superior biocompatibility [11-13]. In this full paper, the surface microstructure characteristics of Fe quantum wires are studied in an aqueous environment, and the interaction mechanism between Fe quantum wires and specific molecules is discussed.

\section{MATERIALS AND METHODS}

\section{Materials}

The Au wire was purchased from Sino-platinum Metals, China, and the Fe wire was purchased from Shenglong Metals, China. The chemicals used were purchased from Sigma-Aldrich, USA, and the ultrapure water $(18.2 \mathrm{M} \Omega . \mathrm{cm})$ was provided with a Millipore system.

\section{Methods}

The device in the present experiment was similar to that in the previous studies from our group [14]. The central section was a self-made electrochemical control unit, which could achieve different functions through programming. A signal generator was used to provide alternating current monitoring signals. The platinum plate and $\mathrm{Ag} / \mathrm{AgCl}$ electrodes were used as counter and reference electrodes, respectively. The fine Fe wire (100 microns in diameter) served as working electrode, on which iron atoms were corroded or deposited.

A key task of the present experiment is to fabricate the atomic-scale Fe junction with quantum conductance. We fabricated such nanostructured materials using two different electrochemical strategies which had been described previously by our group $[15,16]$. The first one was a directly electrochemical corrosion and deposition process [15]. Briefly, the working electrode was first replaced with a fine $\mathrm{Au}$ wire (25 microns in diameter), then it was covered with $\mathrm{AB}$ glue on the slide except the middle part (1 micron in width) was exposed for corrosion. The potential was set at $0.95 \mathrm{~V}$ for corrosion, and an alternating current voltage was simultaneously applied to the wire for monitoring the whole process. With an automatic electrochemical corrosion process, a $\mu \mathrm{m}$-scale Au gap was first formed under the electrolyte solution of $0.5 \mathrm{M} \mathrm{KCl}$. The solution was then replaced with $10 \mathrm{mM} \mathrm{FeCl}_{2}$ solution. By fine-tuning the electrochemical deposition process and its opposite corrosion process, the stepped conductivity change would be clearly shown. This indicated that the junction formed between two gold wires was composed of several Fe atoms, and these atoms were assembled layer by layer with an atomic level. Once the monitoring current reached the set point, the program would automatically cut off the etching/depositon potential, and the atomicscale Fe junction with the preset conductance would be obtained. For avoiding the interference coming from other ions in the solution, the prepared $\mathrm{Fe}$ junction must be transferred to ultra-pure water.

Another electrochemical method for preparing an atomic-scale Fe contact was using a selfterminating electrochemical experiment scheme under the controlling of an external resistance [16]. Briefly, two opposite Fe microelectrodes separated with a micron-scale gap were first formed by cutting the thin Fe wire (100 microns in diameter) which had been glued and insulated with the $A B$ glue on the glass slide. Then, the slide was put into an electrochemical cell containing only pure water. The two Fe microelectrodes formed and an external resistor were placed in series to construct a voltage distribution circuit. The three-electrode electrochemical system was converted into a two-electrode scheme by connecting the reference and counter electrodes. Then, a bias voltage $(\sim 1 \mathrm{~V})$ was applied to the two ends of the circuit. By adjusting the value of external resistance, the atomic-scale Fe junctions with preset conductance values would be obtained. Compared with the first approach, the second one was simpler and easier to control. Moreover, with a pure water environment of the fabrication, the second method avoided the interference of other ions and was more suitable for in-situ experiments.

As described above, the prepared Fe junction would be kept in ultra-pure water. A two-electrode electrochemical scheme would be used to measure conductivity changes, and the voltage was set at $10 \mathrm{mV}$. Once a stable baseline was obtained, the water in the cell was replaced with sample solution of specific concentration, and the following current changes were monitored and recorded continuously by a programmed notebook (current-time curve).

\section{RESULTS AND DISCUSSION}

\section{Interactions of ascorbic acid molecules with $\mathrm{Fe}$ quantum wire}

The purpose of the previous efforts to fabricate atomic-scale junctions was to study the surface 


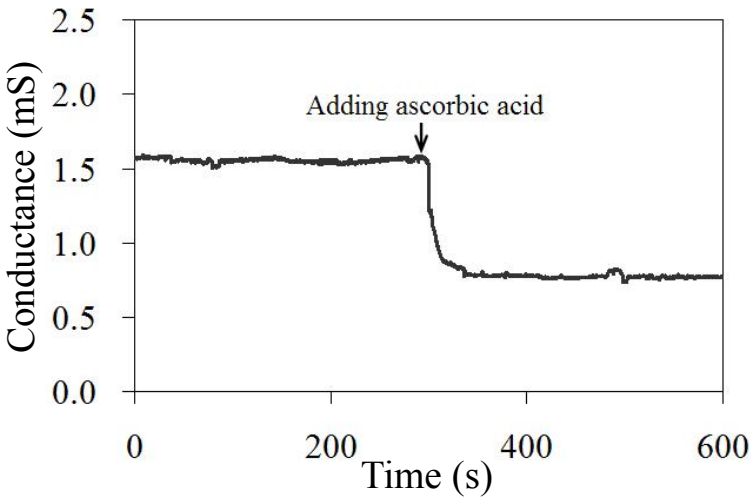

Fig. 1 The conductance response of an atomic-scale Fe junction upon exposure to $10 \mathrm{mM}$ ascorbic acid. The arrow showed the addition of ascorbic acid sample.

properties of the novel nanostructures, so the quantum wire was tested by exposure to the specific molecules. Fig. 1 indicated the conductance change of a representative atomic-scale Fe junction upon exposure to $10 \mathrm{mM}$ ascorbic acid sample. The current flowing through the quantum wire decreased rapidly and then remained constant at a lower value. With lower concentrations, the currents often showed a slower decline process, which indicated a typical process of diffusion control. It must be mentioned that sometimes when we observed upon introduction of ascorbic acid into the cell, the current would drop to zero, showing the breakdown of the quantum wire, which occurred especially for higher concentration solutions. We attributed this phenomenon to the following two reasons. On the one hand, diffusion and adsorption of several molecules onto quantum wires are not easy to control with the present experiment. On the other hand, with an atomic level study, the atomic configuration changes of the quantum wire upon adsorption of sample molecules would not always be consistent with macro expectations that had also been found by other groups $[17,18]$.

\section{Conductance mechanism}

The behavior of the conductance change with time upon introduction of ascorbic acid was worthy of comment. Although up to now, the research on quantum elastic regime was not perfect, it was generally believed that the decrease in conductivity might be due to the interfacial adsorbate scattering for the conductive electrons in the quantum wire $[19,20]$. Furthermore, the change of conductance produced by adsorption depended on the binding force of molecules on quantum wires $[21,22]$. The above experiments showed that upon exposing the quantum wire to ascorbic acid, the conductance often had a big drop ( $>50 \%$ ), even to zero, which indicated that there was a strong interaction between ascorbic acid and Fe quantum wire. In the previous studies of our group on surface structure of atomic-scale Ag junction in solution [10], we believed that there were some Ag ions in the surface microstructures of $\mathrm{Ag}$ quantum wires and these $\mathrm{Ag}$ ions brought about many novel surface phenomena of Ag quantum wire. As we had known, iron was a more active metal than silver, so we assumed that, like Ag quantum wires, there were also some metal ions in the surface microstructure of atomic-scale $\mathrm{Fe}$ junction in aqueous solution. The following studies focused on whether these metal ions are iron(II) or iron(III) ions. Because ascorbic acid was well known for its reducing ability, it was able to easily reduce $\mathrm{Fe}^{3+}$ to $\mathrm{Fe}^{2+}$ [23]. For a metal quantum wire, this surface reduction process was equivalent to a charge injection process. If these metal ions on the surface of Fe quantum wire were iron(III) ions, the process of conductance increasing should be observed. But as shown in Fig. 1, the observed results were contrary, which indicated that it was not reasonable to assume the existence of iron(III) ions in the surface microstructures of atomic-scale Fe junction. Furthermore, the previous study by Jacobsen and Scheers et al showed that the ascorbic acid could form a complex with iron(II) ions and the complexation ability of ascorbic acid towards $\mathrm{Fe}^{2+}$ was strong $[23,24]$, which indicated that there might be iron(II) ions on the surface of the Fe quantum wire and the interactions between these iron(II) ions and ascorbic acid resulted in a great change in the conductance of the atomic-scale Fe junction. We also noticed another work done by $\mathrm{Xu}$ and coworkers [25], who studied the interactions between dopamine and iron(III) ions with a microcantilever and observed the dopamine and iron(III) ions interacted strongly, but not with iron(II) ions. Therefore, we studied the effect of dopamine adsorption on the conductivity of Fe quantum wire. Fig. 2a showed the conductance of the Fe junction does not change significantly upon the addition of dopamine. In addition, the adsorption showed no obvious concentration dependence. This further indicated that the metal ions in the surface microstructure of the atomic-scale Fe junction are not iron(III) ions.

In order to study this problem in more detail, two further experiments were carried out. The first one was studying the effect of adsorption on 

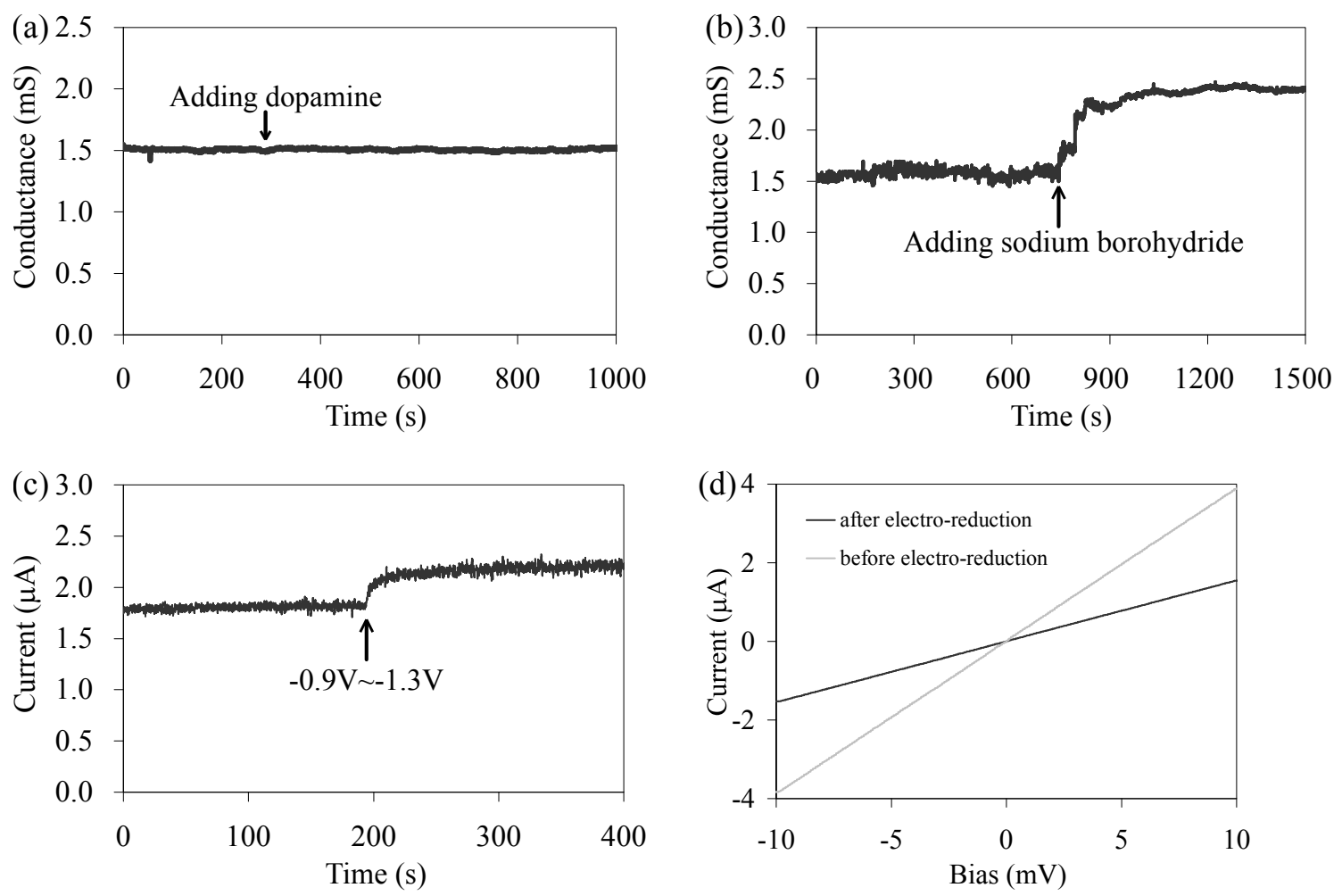

Fig. 2 Representative conductance response of a Fe quantum wire when it interacted with dopamine (a) and sodium borohydride (b). (c) Representative response of AC monitoring current versus time when the equilibrium potential $(\sim-0.9 \mathrm{~V})$ applied to the Fe quantum wire was converted to the reduction potential $(\sim-1.3 \mathrm{~V})$. (d) I-V characteristics of a Fe quantum wire before and after it was electrochemically reduced.

conductance of Fe junction by substituting ascorbic acid with sodium borohydride. The sodium borohydride was chosen because it was a good chemical reducing agent and was widely used to reduce the iron(II) ions to prepare Fe nanoparticles and nanochains [26-28]. Fig. 2b showed the addition of sodium borohydride resulted in a significant conductance increasing process. The concentration dependence of the conductance changing for the quantum wire in sodium borohydride solution is similar to that of ascorbic acid. With a lower concentration, the conductance showed a slower changing process, but the relative change $(\Delta \mathrm{G} / \mathrm{G})$ showed no obvious dependence on the concentration. This might be due to the reduction of iron(II) ions in the surface microstructure of Fe quantum wires to Fe atoms, which increased the conductivity. The second one was conducting an electrochemical reduction process. The fabricated Fe quantum wires were used as working electrodes in electrochemical cell, in which a Pt slice and an $\mathrm{Ag} / \mathrm{AgCl}$ electrode were used as counter and reference electrodes, respectively, and only the pure water was used as electrolyte.
An alternating current (AC) generator was used to generate AC signal $(\sim 13 \mathrm{mV}, 10 \mathrm{~Hz})$, which was used to monitor the conductance changing of the Fe quantum wire. To avoid short circuit damage to signal source, an additional resistance $(1 \mathrm{k} \Omega$ ) was connected to the circuit. The equilibrium potential $(\sim-0.9 \mathrm{~V})$ was initially applied to the Fe quantum wire for forming a steady baseline. It was then converted to the reduction potential $(\sim-1.3 \mathrm{~V})$. During the process, the current could be observed to rise and stabilize at a higher value (Fig. 2c). This might be due to the electro-reduction of iron(II) ions to Fe atoms on the surface of Fe quantum wire, which increased the conductivity of Fe quantum wire. Fig. $2 d$ showed the I-V curves of Fe quantum wires before and after electroreduction. In contrast, we repeated the above two experiments with a thin bulk Fe wire (100 $\mu \mathrm{m}$ in diameter) instead of the Fe quantum wire. No current changes had been observed, which illustrated the unique properties of the Fe quantum wire different from the bulk one. The above experimental data further supported the mechanism we had proposed. 


\section{CONCLUSION}

To summarize, we had investigated the surface structural characteristics of $\mathrm{Fe}$ quantum wires in solution through the conductivity changes of atomicscale $\mathrm{Fe}$ junction caused by their interaction with specific molecules. The conductivity of Fe junction revealed a decreasing process and almost no significant change when it was exposed to ascorbic acid and dopamine molecules, respectively. Based on the above experimental data and relevant results from literature reports, we thought there were some iron(II) ions in the surface microstructure of a newly fabricated Fe quantum wire, not iron(III) ions. This view was further confirmed by two other experiments. The first one was that when the Fe quantum wire interacted with the chemical reductant sodium borohydride, the conductance showed a process of increasing to a stable value. The second one was that when the equilibrium potential applied to the $\mathrm{Fe}$ quantum wire was converted to the reduction potential, the conductance of the Fe quantum wire showed a similar process as that of the previous experiment. This study enabled us to better understand the surface microstructure characteristics of the magnetic material $\mathrm{Fe}$ quantum wires in solution and was meaningful for materials science, life science and chemical and biological sensing applications.

Acknowledgements: The authors thank the Hebei Provincial Foundation for Returness (C201803) and the Hebei Provincial Natural Science Foundation of China (B2015201161) for financial support.

\section{REFERENCES}

1. Ai Y, Smida H, Ghilane J, Vila N, Ghanbaja J, Walcarius A, Lacroix JC (2017) Copper nanowires through oriented mesoporous silica: a step towards protected and parallel atomic switches. Sci Rep 7, ID 17752.

2. Mekseriwattana W, Srisuk S, Tantiapibalkun Y, Prapainop K (2019) Preparation of superparamagnetic iron oxide nanoparticles and investigation of their interaction with cells. ScienceAsia 45, 60-64.

3. Linetskiy I, Starcukova J, Hubalkova H, Starcuk Z, Ozcan M (2019) Evaluation of magnetic resonance imaging issues of titanium and stainless steel brackets. ScienceAsia 45, 145-153.

4. Hwang TW, Branagan SP, Bohn PW (2013) Chemical noise produced by equilibrium adsorption/desorption of surface pyridine at $\mathrm{Au}-\mathrm{Ag}$ - $\mathrm{Au}$ bimetallic atomscale junctions studied by fluctuation spectroscopy. $J$ Am Chem Soc 135, 4522-4528.

5. Shi P, Bohn PW (2010) Electrochemical control of stability and restructuring dynamics in $\mathrm{Au}-\mathrm{Ag}-\mathrm{Au}$ and
Au-Cu-Au bimetallic atom-scale junctions. ACS Nano 4, 2946-2954.

6. Hwang TW, Bohn PW (2011) Robust Au-Ag-Au bimetallic atom-scale junctions fabricated by selflimited $\mathrm{Ag}$ electrodeposition at Au nanogaps. ACS Nano 5, 8434-8441.

7. Staiger T, Wertz F, Xie FQ, Heinze M, Schmieder P, Lutzweiler C, Schimmel T (2018) Macro-mechanics controls quantum mechanics: mechanically controllable quantum conductance switching of an electrochemically fabricated atomic-scale point contact. Nanotechnology 29, ID 025202.

8. Zhao ZK, Liu R, Mayer D, Coppola M, Sun L, Kim YS, Wang CK, Ni LF, et al (2018) Shaping the atomicscale geometries of electrodes to control optical and electrical performance of molecular devices. Small 14, ID 1703815.

9. Duan BK, Zhang JY, Bohn PW (2012) Conductancebased chemical sensing in metallic nanowires and metal-semiconductor nanostructures. Anal Chem 84, 2-8.

10. Dong XD, Liu JH, Zhang BL, Xia Y (2012) Surface structural analysis of electrochemically fabricated $\mathrm{Ag}$ quantum wire by its interactions with $\mathrm{NH}_{3}$ molecules in an aqueous environment. Electrochim Acta 74, 78-82.

11. Song SQ, Bohuslav G, Capitano A, Du J, Taniguchi K, Cai ZH, Sun L (2012) Experimental characterization of electrochemical synthesized Fe nanowires for biomedical applications. J Appl Phys 111, ID 056103.

12. Hu J, Zhang F, Wang J, Xiao JQ (2015) Synthesis of single-crystalline Fe nanowires using catalystassisted chemical vapor deposition. Mater Lett 160, 529-532.

13. Weissmann M, Errico L (2013) Effect of low dimensionality and encapsulation on the magnetic and hyperfine properties of iron nanowires. J Phys Chem C 117, 19684-19688.

14. Dong XD, Xia Y, Zhu GY, Zhang BL (2007) Molecular sensing with the tunnel junction of an Au nanogap in solution. Nanotechnology 18, ID 395502.

15. Liu JH, Zheng P, Zhang QY, Dong XD (2015) Electrochemical fabrication of iron quantum wire with atomic-scale. Asian J Chem 27, 2320-2322.

16. Zheng P, Zhu J, Liu JH, Dong XD, Xia Y (2018) Study of the electrochemical preparation of atom-scale iron quantum wire controlled by an external resistor. Int J Electrochem Sci 13, 7571-7577.

17. Xiang J, Liu B, Liu B, Ren B, Tian ZQ (2006) A selfterminated electrochemical fabrication of electrode pairs with angstrom-sized gaps. Electrochem Commun 8, 577-580.

18. Li CZ, Bogozi A, Huang W, Tao NJ (1999) Fabrication of stable metallic nanowires with quantized conductance. Nanotechnology 10, 221-223. 
19. Bogozi A, Lam O, He HX, Li C, Tao NJ (2001) Molecular adsorption onto metallic quantum wires. $J$ Am Chem Soc 123, 4585-4590.

20. Li CZ, He HX, Bogozi A, Bunch JS, Tao NJ (2000) Molecular detection based on conductance quantization of nanowires. Appl Phys Lett 76, 1333-1335.

21. He HX, Shu C, Li CZ, Tao NJ (2002) Adsorbate effect on the mechanical stability of atomically thin metallic wires. $J$ Electroanal Chem 522, 26-32.

22. He HX, Tao NJ (2002) Interactions of molecules with metallic quantum wires. Adv Mater 14, 161-164.

23. Jacobsen C, Adler-Nissen J, Meyer AS (1999) Effect of ascorbic acid on iron release from the emulsifier interface and on the oxidative flavor deterioration in fish oil enriched mayonnaise. J Agric Food Chem 47, 4917-4926.

24. Scheers N, Andlid T, Alminger $M$, Sandberg AS (2010) Determination of $\mathrm{Fe}^{2+}$ and $\mathrm{Fe}^{3+}$ in aqueous solutions containing food chelators by differential pulse anodic stripping voltammetry. Electroanalysis 22, 1090-1096.

25. Xu YM, Zhang BL, Wu SH, Xia Y (2009) The adsorption of dopamine on gold and its interactions with iron(III) ions studied by microcantilevers. Anal Chim Acta 649, 117-122.

26. Tang HZ, Zhan XT, Wu Z, Du Y, Talbi A, Pernod $P$ (2015) Controllable and facile fabrication of $\mathrm{Fe}$ nanoparticles/nanochains and their magnetic properties. J Magn Magn Mater 377, 472-476.

27. Tang HZ, Han D (2017) Controllable preparation of iron nanostructures and their magnetic properties. $J$ Magn Magn Mater 444, 125-131.

28. Zhan XT, Tang HZ, Du Y, Talbi A, Zha JL, He $\mathrm{JH}$ (2013) Facile preparation of $\mathrm{Fe}$ nanochains and their electromagnetic properties. RSC Adv 3, 15966-15970. 